\title{
Mediator MED23 plays opposing roles in directing smooth muscle cell and adipocyte differentiation
}

\author{
Jing-wen Yin, ${ }^{1}$ Yan Liang, ${ }^{1}$ Ji Yeon Park, ${ }^{2}$ Dongrui Chen, ${ }^{3}$ Xiao Yao, ${ }^{1}$ Qi Xiao, ${ }_{4}^{4}$ Zhen Liu, ${ }^{1}$ Bo Jiang, ${ }^{1,7}$ \\ Yu Fu, ${ }^{1}$ Menghan Bao, ${ }^{1}$ Yan Huang, ${ }^{1}$ Yuting Liu, ${ }^{5}$ Jun Yan, ${ }^{5}$ Min-sheng Zhu, ${ }^{4}$ Zhongzhou Yang, ${ }^{4}$ \\ Pingjin Gao, ${ }^{3}$ Bin Tian, ${ }^{2}$ Dangsheng $\mathrm{Li}^{6}{ }^{6}$ and Gang Wang ${ }^{1,8}$ \\ ${ }^{1}$ State Key Laboratory of Cell Biology, Institute of Biochemistry and Cell Biology, Shanghai Institutes for Biological Sciences, \\ Chinese Academy of Sciences, Shanghai 200031, China; ${ }^{2}$ Department of Biochemistry and Molecular Biology, New Jersey \\ Medical School, University of Medicine and Dentistry of New Jersey, Newark, New Jersey 07103, USA; ${ }^{3}$ Laboratory of Vascular \\ Biology, Key Laboratory of Stem Cell Biology, Institute of Health Sciences, Shanghai Institutes for Biological Sciences, Chinese \\ Academy of Sciences, Shanghai 200025, China; ${ }^{4}$ MOE Key Laboratory of Model Animal for Disease Study, Model Animal \\ Research Center, Nanjing University, Nanjing 210061, China, ${ }^{5}$ Key Laboratory of Computational Biology, CAS-MPG Partner \\ Institute for Computational Biology, Shanghai Institutes for Biological Sciences, Chinese Academy of Sciences, Shanghai 200031, \\ China; ${ }^{6}$ Shanghai Information Center for Life Sciences, Shanghai Institutes for Biological Sciences, Chinese Academy of \\ Sciences, Shanghai 200031, China
}

The Mediator complex functions as a control center, orchestrating diverse signaling, gene activities, and biological processes. However, how Mediator subunits determine distinct cell fates remains to be fully elucidated. Here, we show that Mediator MED23 controls the cell fate preference that directs differentiation into smooth muscle cells (SMCs) or adipocytes. Med23 deficiency facilitates SMC differentiation but represses adipocyte differentiation from the multipotent mesenchymal stem cells. Gene profiling revealed that the presence or absence of Med23 oppositely regulates two sets of genes: the RhoA/MAL targeted cytoskeleton/SMC genes and the Ras/ELK1 targeted growth/ adipogenic genes. Mechanistically, MED23 favors ELK1-SRF binding to SMC gene promoters for repression, whereas the lack of MED23 favors MAL-SRF binding to SMC gene promoters for activation. Remarkably, the effect of MED23 on SMC differentiation can be recapitulated in zebrafish embryogenesis. Collectively, our data demonstrate the dual, opposing roles for MED23 in regulating the cytoskeleton/SMC and growth/adipogenic gene programs, suggesting its "Ying-Yang" function in directing adipogenesis versus SMC differentiation.

[Keywords: Mediator complex; MED23; MAL; ELK1; smooth muscle cell; adipocyte; differentiation]

Supplemental material is available for this article.

Received March 26, 2012; revised version accepted August 13, 2012.

Cell fate determination in metazoans is precisely controlled by sophisticated spatiotemporal patterns of RNA polymerase II (Pol II)-mediated transcription in response to various intracellular and extracellular signals. Genetic or environmental alterations that perturb the regulation of transcription can alter cell fate specifications, leading to a variety of developmental defects. Control over lineagespecific transcriptional programs has generally been attributed to the specific DNA-binding transcription factors. For example, PPAR $\gamma$ controls the adipocyte program (Tontonoz et al. 1995), MyoD controls the skeletal muscle

\footnotetext{
${ }^{7}$ Present address: Department of Cardiovascular and Thoracic Surgery, Affiliated Hospital of Xuzhou Medical College, Xuzhou 221002, China. ${ }^{8}$ Corresponding author

E-mail gwang22@sibs.ac.cn

Article published online ahead of print. Article and publication date are online at http://www.genesdev.org/cgi/doi/10.1101/gad.192666.112.
}

program (Olson 1990), and Runx2 controls the osteocyte program (Komori 2002). Most strikingly, four "Yamanaka" factors-Oct4, Sox2, c-Myc, and Klf4 — can directly reprogram many types of somatic cells into pluripotent stem cells (Takahashi and Yamanaka 2006; Wernig et al. 2008). The molecular mechanisms of how these master regulatory transcription factors function have not been fully elucidated.

To ensure precise transcriptional control of cell fate determination and development, the master transcription factors are subjected to further regulatory control. Eukaryotes have evolved elaborate transcriptional machinery consisting of multiple cofactors/cofactor complexes to modulate the basal transcriptional apparatus. Two recent studies demonstrated that the cofactors control the cell fate by modulating the distinct transcription factors. First, TAZ coactivates Runx2-dependent 
transcription during osteocyte differentiation while repressing PPAR $\gamma$-dependent adipogenesis transcription (Hong et al. 2005). Another cofactor, PRDM16, has been observed to interact with PPAR $\gamma$ and C/EBP $\beta$ to initiate the brown fat program while repressing the skeletal muscle program (Seale et al. 2008; Kajimura et al. 2009). The Mediator complex interacts directly with various transcription factors and Pol II to regulate the transcription of protein-coding genes. The list of documented signaling and transcription factors known to impinge on the Mediator complex is ever expanding, including TGF $/$ SMADs, MAPK/ELK1, Hh/Gli, lipid/SREBP, and multiple hormones/ nuclear receptors (Blazek et al. 2005). Through the interaction between transcription factors and the Mediator complex, Pol II recruitment is significantly enhanced, and transcriptional complexes at the particular gene promoters are stabilized. In this capacity, the Mediator complex serves as a control center for integrating environmental and developmental cues for regulating diverse physiological processes, including cell growth and differentiation, pluripotency maintenance, and homeostasis (Malik and Roeder 2010). For example, the MED1 subunit of the Mediator complex is a well-established target for a variety of nuclear receptors (Malik and Roeder 2010), and fibroblasts isolated from $\mathrm{Med1} 1^{-/-}$embryos have been found to exhibit a selective loss of the PPAR $\gamma$-dependent ability to differentiate into adipocytes (Ge et al. 2002). The MED12 subunit has also been shown to repress neuronal gene expression in nonneuronal cells through epigenetic gene silencing (Ding et al. 2008). Furthermore, the MED15 subunit has been shown to induce axis duplication and mesendoderm differentiation in Xenopus embryos (Kato et al. 2002). Thus, growing evidence suggests that distinct Mediator components seem to be specific in directing a particular cell fate or development process; however, an example that one subunit can control a molecular switch between two cell fates has not been documented.

The MED23 subunit was originally identified as a target of the viral oncoprotein E1A (Boyer et al. 1999). Although MED23 has been proved to be essential to link insulin signaling to the adipogenesis transcription cascade (Wang et al. 2009), its function in development has not been fully elucidated, as Med23 knockout embryos died at approximately day 10.5 of gestation (Balamotis et al. 2009). Med23 deficiency prevents adipocyte differentiation by attenuating the expression of an insulin-stimulated immediateearly gene, krox20/Egr2 (Wang et al. 2009); however, the cell fate with Med23 deficiency remains unknown. To explore the cellular and molecular consequence of Med23 deficiency, we carried out further investigations by examining whether Med23 can regulate commitment of mesenchymal cells to different lineages. In this study, we first observed that the morphology of the knockout mouse embryonic fibroblasts (MEFs) was enlarged and flattened, and the expression levels of RhoA/MAL-regulated cytoskeleton/smooth muscle cell (SMC) genes were greatly increased in Med23 knockout MEFs. Consistently, we found that Med23 deficiency also promotes differentiation of mesenchymal stem cells into SMCs while inhibiting the differentiation of these cells into adipocytes.
Moreover, this phenotype was recapitulated during zebrafish development. At the molecular level, the presence of MED23 favors the binding of the ELK1-SRF complex to the SMC genes to repress their expression, whereas the absence of MED23 favors MAL-SRF complex formation, which subsequently activates SMC gene expression. These results suggest that MED23 functions as a molecular switch between the Ras and RhoA pathways that controls differentiation into adipocytes or SMCs. Therefore, our study provides an example of how a transcription cofactor can have a "Ying-Yang" role in determining two distinct cell fates. Our results also improve our understanding of the intricate molecular regulation of SMC and adipocyte development, which should be beneficial for elucidating the molecular basis of both obesity-related and SMC-related diseases.

\section{Results}

Med23 deficiency promotes cytoskeleton gene
expression

Med23 knockout mice display embryonic lethality, which is likely caused by systematic circulatory failure (Balamotis et al. 2009). To further understand the role of MED23 in development and gene regulation, we analyzed the morphology of the MEFs derived from wild-type and Med23 knockout embryos. Knockout MEFs are larger and more flattened than wild-type MEFs and exhibited an increased number of stress fibers and focal adhesions, as revealed by phalloidin and anti-paxillin antibody staining (Fig. 1A). The protein levels of the cytoskeleton genes Vincullin (Vcl), Acta2, and Actg2 and their critical regulator, SRF, were significantly increased in knockout MEFs (Fig. 1B). Single or multiple copies of serum response elements (SREs) exist in the promoters of these cytoskeletonrelated genes and bestow serum responsiveness (Miano 2003; Buchwalter et al. 2004; Vartiainen et al. 2007). Indeed, these cytoskeleton genes in both wild-type and Med23 knockout MEFs showed a significant increase in expression after the addition of serum (Fig. 1C). However, their responsiveness in knockout MEFs was much greater than that of wild-type MEFs (Fig. 1C). Reporter plasmids with five tandem copies of SREs (CArG boxes) or the SREcontaining Sm22a promoter sequence also showed greater serum response and a significant increase in knockout cells compared with wild-type cells (Fig. 1D). To rule out the possibility that this effect on gene expression was due to the adaptive change of the wild-type and knockout MEFs, the human Med23 (hMed23) gene was reintroduced into Med23 knockout MEFs using retroviral transduction (Fig. 1G). An anti-CDK8 antibody was able to pull down representative Mediator subunits, including MED6 and MED16, as well as the ectopically expressed hMED23, indicating the proper expression and incorporation of ectopic hMED23 into the functional Mediator complex (Supplemental Fig. S1B). Reintroducing hMed23 into the knockout MEFs rescued the morphology phenotype to resemble the wild-type MEFs, at least partially, under both the subconfluent state and the confluent state (Supple- 
A
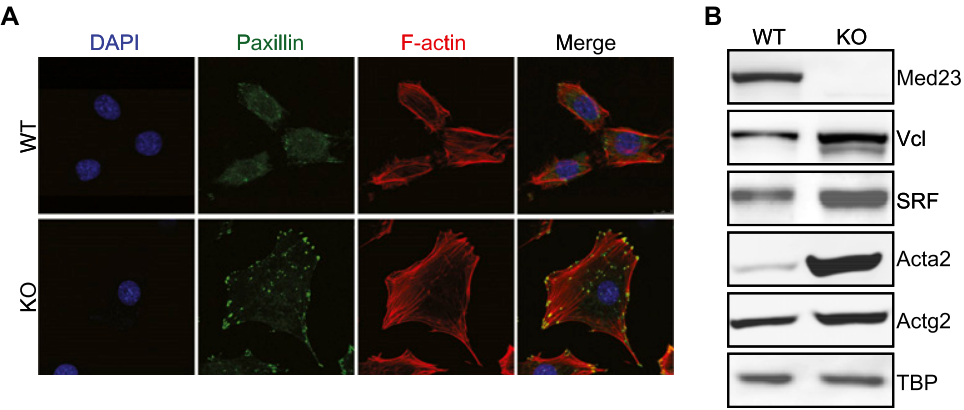

C
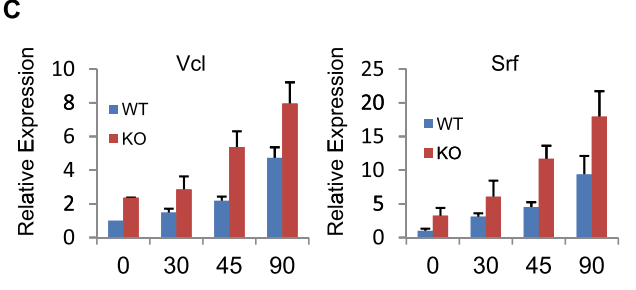

D
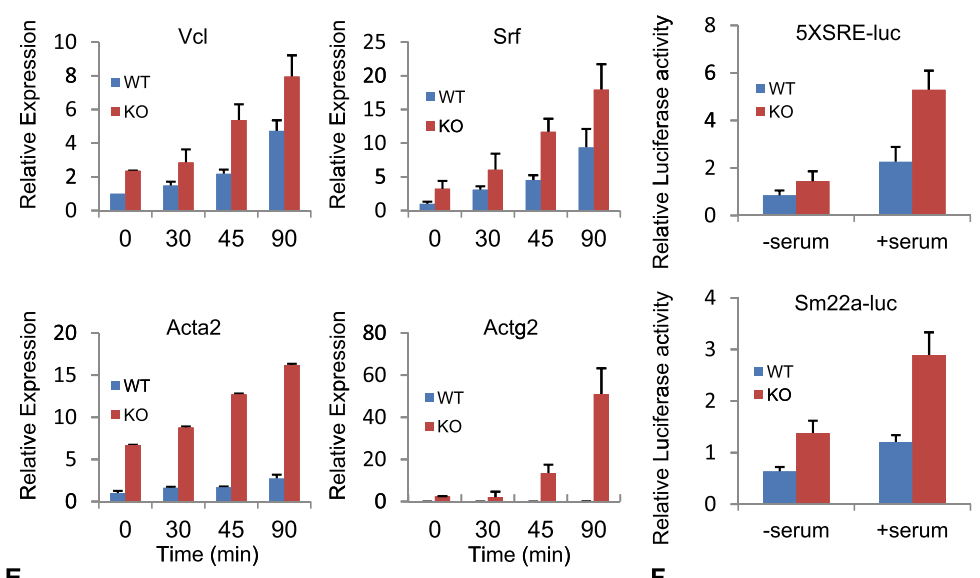

E
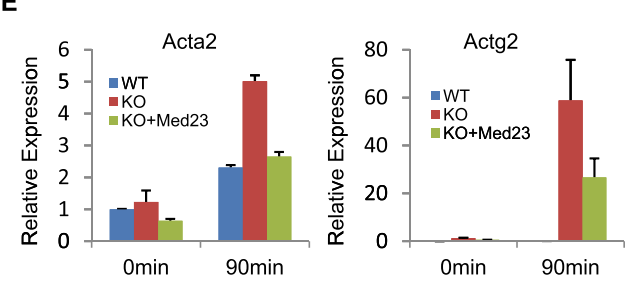
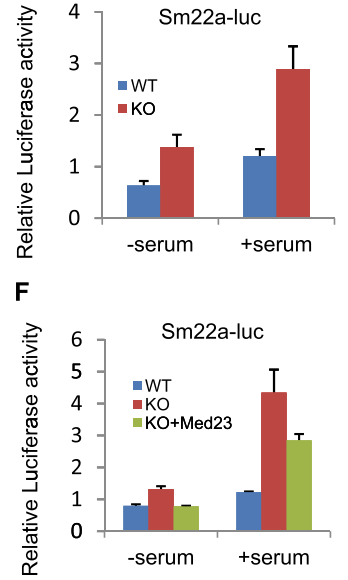

Figure 1. Analysis of the cytoskeleton gene expression in wild-type (WT) and Med23 knockout (KO) MEFs. (A) Wild-type and Med23 knockout MEFs were maintained at steady state $(10 \%$ FBS), fixed, and stained with DAPI, phalloidin, and anti-paxillin antibody, respectively. Bar, $10 \mu \mathrm{m}$. $(B)$ The total protein were extracted from wild-type and Med23 knockout MEFs and analyzed by Western blot using the indicated antibodies. TATA-box-binding protein (TBP) was blotted as a loading control. $(C)$ Wild-type and Med23 knockout MEFs were serum-starved in $0.5 \%$ FBS and then stimulated with $15 \%$ FBS. At different time points after stimulation, total RNA were extracted and subjected to real-time PCR analysis of Vcl, Srf, Acta2, and Actg2. The expression was normalized to Gapdh. (D) Wild-type and Med23 knockout MEFs were transfected with 5XSRE-luc or Sm22a-luc reporter plasmids. After maintenance in $0.5 \%$ FBS for $24 \mathrm{~h}$ and stimulation with $15 \%$ FBS for an additional $12 \mathrm{~h}$, luciferase activity was measured. (E) Med23 knockout MEFs were infected with retroviruses expressing hMed23 (KO+Med23) and then selected for puromycin resistance. Wild-type, knockout, and KO+Med23 MEFs were serum-starved or serum-simulated as in $C$. Total RNA were prepared at the indicated time points and analyzed using real-time PCR. The expression was normalized to Gapdh level. (F) Sm22a-luc reporter was transfected into wild-type, knockout, and KO+Med23 MEFs and serum-starved or serum-stimulated as in $D .(G)$ The total protein was extracted from wild-type, knockout, and $\mathrm{KO}+\mathrm{Med} 23$ MEFs and analyzed by Western blot using antibodies against MED23 and ACTA2. TBP was blotted as a loading control.

G

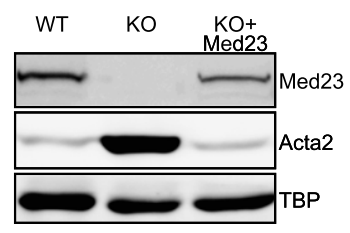

mental Fig. S1A). Moreover, ectopic hMED23 significantly rescued (i.e., repressed) the up-regulated mRNA, protein, and reporter levels of cytoskeleton genes (Fig. 1E-G), indicating the specific causal relationship between Med23 deficiency and the up-regulation of cytoskeleton gene.

In order to assess the specificity of the Mediator components in regulating these cytoskeleton genes, viralmediated knockdown of Med1, Med12, Med14, Med23, Med28, or Cyclin C (CycC) was performed in immortalized wild-type MEFs. The knockdown efficiency was confirmed by Western blot or real-time PCR analysis (Fig 2A; Supplemental Fig. S1C,D). Strikingly, only Med23 (Med23specific siRNA oligo [siMed23]) but not other subunit deficiency increased the cytoskeleton gene expression (Supplemental Fig. S1C,D). Collectively, these results strongly suggest that the expression of serum-responsive cytoskeleton genes is selectively up-regulated by the de- pletion of MED23. Therefore, MED23 seems to play a specific restrictive role in cytoskeleton gene expression.

\section{Med23-regulated cytoskeleton genes are controlled by the RhoA/MAL pathway}

RhoA signaling is a critically important pathway for controlling cytoskeleton gene expression during serum stimulation (Settleman 2003; Posern and Treisman 2006). Treatment of cells with the RhoA inhibitor LatB not only suppressed the serum responsiveness, but also suppressed the up-regulated $\mathrm{Vcl}$ and Srf mRNA expression in siMed23 MEFs to the same extent as in control-specific siRNA oligo (siCtrl) MEFs (Fig. 2B), suggesting that RhoA signaling is required for the increased cytoskeleton gene expression upon Med23 depletion. We then investigated how Med23 deficiency could affect the RhoA signaling 

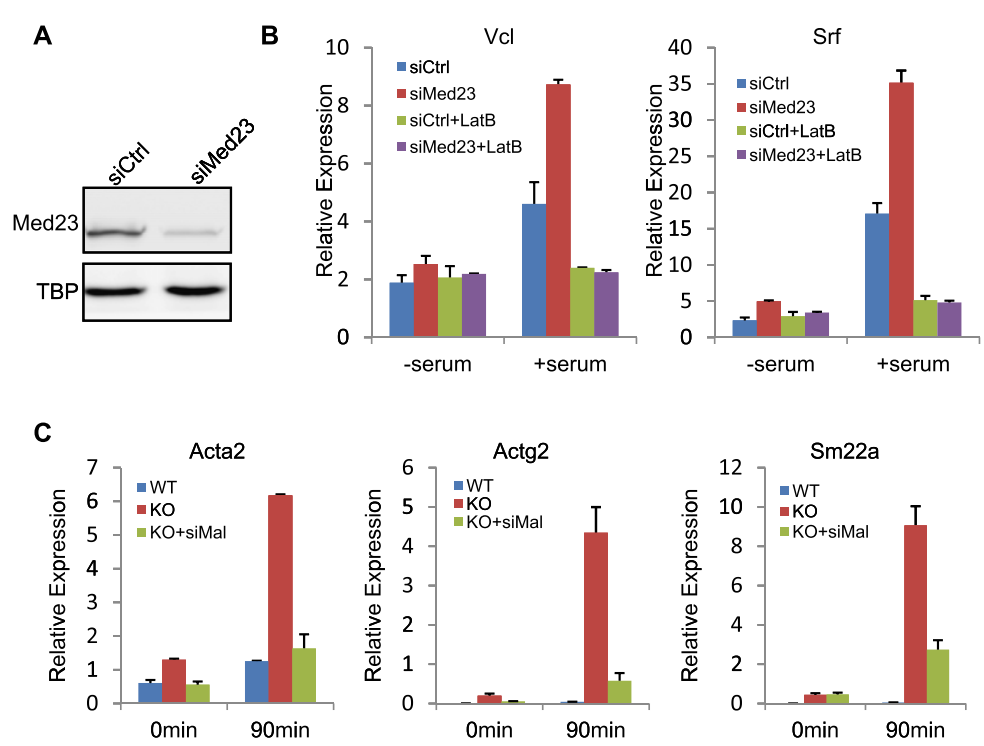

D

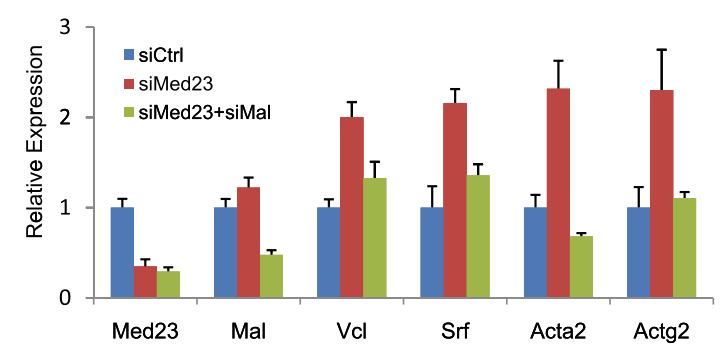

Figure 2. Med23 deficiency up-regulates cytoskeleton genes via the RhoA/MAL pathway. (A) Stable MEF cell lines were generated by retroviral expression of siMed23 or siCtrl. After puromycin selection, the knockdown efficiency was analyzed by Western blot. (B) siCtrl and siMed23 MEFs were serum-starved or serum-stimulated with or without LatB pretreatment. The expression of $\mathrm{Vcl}$ and $\mathrm{Srf}$ was examined by realtime PCR and normalized to Gapdh level. $(C)$ Med23 knockout (KO) MEFs were infected with retroviruses expressing siMal (KO+siMal). After serum starvation or stimulation as in Figure 1C, total RNA was extracted and subjected to real-time analysis of Acta2, Actg2, and Sm22a expression. Their expression was normalized to Gapdh. $(D)$ siMed23 MEFs were infected with retroviruses expressing siMal (siMed23+siMal). The expression of cytoskeletal genes ( $V c l, S r f, A c t a 2$, and Actg2) was analyzed in serum-stimulated siCtrl, siMed23, and siMed23+siMal MEFs by real-time PCR. The expression was normalized to Gapdh. pathway. Surprisingly, Med23 deficiency did not increase the amount of active RhoA in Med23 knockout MEFs, as indicated by a RhoA-GTP pull-down experiment, but even decreased it somewhat (Supplemental Fig. S2A). Additionally, phosphorylation of MYL9, which has been shown to be a downstream event in response to RhoA activation (Van Aelst and D'Souza-Schorey 1997), was not induced by the absence of MED23, although the total MYL9 level was significantly up-regulated (Supplemental Fig. S2B).

We looked into the further downstream events of the RhoA signaling pathway. Upon RhoA activation via actin polymerization, the G-actin-bound transcription factor MAL is released from the cytoplasm into the nucleus and forms a complex with SRF, thus activating gene expression (Miralles et al. 2003; Posern and Treisman 2006). Mal has two other family members, Myocardin (Myocd) and Mkl2 (Cen et al. 2004); however, Mal was expressed at a level that was $>13$-fold higher than the other family members in MEFs (Supplemental Fig. S2C), which makes it the major factor controlling cytoskeleton gene expression. We established cell lines by using retroviral-mediated siRNA to knock down Mal expression (siMal) in Med23 knockout MEFs (KO+siMal), and this resulted in the repression of the up-regulated cytoskeleton genes in Med23 knockout MEFs, as assessed by the expression of Acta2, Actg2, and Sm22a (Fig. 2C). An effect similar to that in the Med23 knockout MEFs was also observed in siMed23
MEFs (Fig. 2D). These results suggest that up-regulation of the cytoskeleton genes is through MAL.

Next, we examined whether Med23 deficiency has any effect on the transcriptional activity of MAL. Transient transfection assays were performed using Gal4-Mal or Gal4-Malc (C-terminal activation domain) plasmids. Although the activity of Gal4-Malc was more robust than that of Gal4-Mal, both fusion proteins activated transcription at similar levels in wild-type and knockout MEFs (Supplemental Fig. S2D), which rules out the possibility that MED23 directly affects the activity of the MAL activation domain when it is taken away from the endogenous promoter context.

SRF is a necessary cofactor for cytoskeleton gene expression. Both the protein and mRNA levels of SRF were slightly up-regulated in knockout MEFs (Fig. 1B,C); however, unlike MAL, SRF failed to increase Sm22a-luc activity in a dosage-dependent manner (Supplemental Fig. S2E). We also overexpressed SRF in wild-type MEFs. Although ectopic SRF expression in wild-type MEFs was much more than the endogenous SRF in knockout MEFs, it failed to significantly increase the cytoskeleton gene expression represented by $\mathrm{Vcl}$ and Myl9 (Supplemental Fig. S2F,G). Collectively, these observations suggest that the modest SRF increase cannot account for the drastic increase in cytoskeleton gene expression.

Overall, our data so far indicate that Med23 deficiency does promote the serum-responsive, RhoA/MAL targeted 
Yin et al.

cytoskeleton gene expression, although it does not alter RhoA or MAL activities per se. However, as we further reveal in the following sections, there exists a sophisticated mechanism by which MED23 modulates the dynamic cross-competition between the RhoA/MAL and MAPK/ELK1 pathways to direct distinct gene programs and cell fates.

\section{Med23 deficiency up-regulates a set of RhoA/MAL targeted cytoskeleton/smooth muscle genes and down-regulates a set of Ras/ELK1 targeted growth/adipogenic genes}

It is well-known that SRF is the indispensable cofactor for RhoA/MAL-controlled expression of the cytoskeleton genes and Ras/ELK1-controlled early growth genes (Miano 2003; Posern and Treisman 2006). We checked how broadly Med23 deficiency impacts the SRF target genes. Previously, all direct SRF target genes were identified through overexpression of SRF-VP16 in $\mathrm{Srf}^{-/-}$cells (Philippar et al. 2004). These genes were clustered according to their biological functions into muscle lineages, growthrelated, and other processes. We performed microarray analysis of wild-type and Med23 knockout MEFs in the presence or absence of serum stimulation (for 30 and 90 min). Self-organizing map (SOM) clustering of SRF target genes with reliable signals yielded two different clusters, which are oppositely affected by Med23 knockout (cluster 1, generally up-regulated; cluster 2, generally downregulated) (Fig. 3A). Strikingly, 10 muscle lineage genes (out of 11) were enriched in cluster 1 , while five growthrelated genes (out of eight) were enriched in cluster 2 (Fig. $3 \mathrm{~A})$. Interestingly, many muscle lineage and growth-related genes have been shown to be regulated by the RhoA/ MAL or Ras/ELK1 pathway, respectively (Gineitis and Treisman 2001; Miano 2003; Buchwalter et al. 2004). These results suggested that these two distinct sets of SRF target genes respond oppositely to Med23 depletion.
A

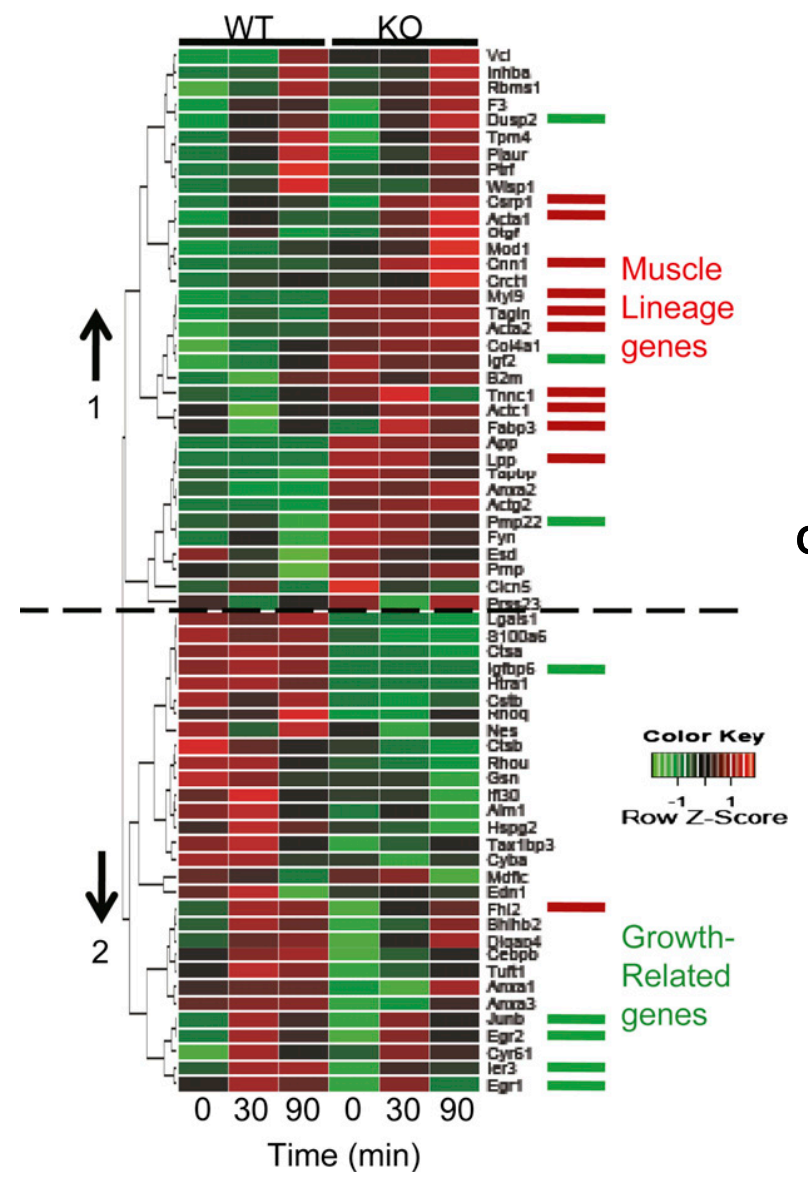

B
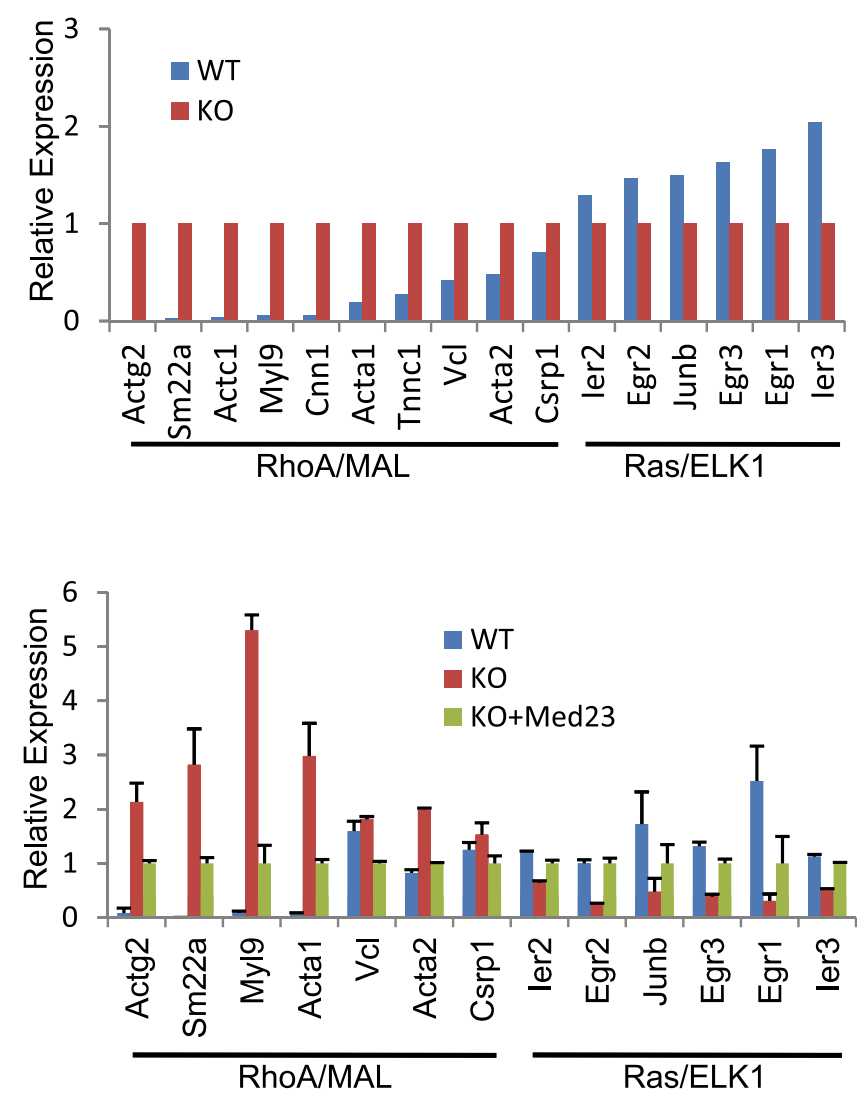

Figure 3. The effect of Med23 deficiency on SRF target gene expression. (A) Gene profiling of wild-type (WT) and Med23 knockout MEFs with or without serum induction (30 and $90 \mathrm{~min}$ ). The degree of induction is reflected by relative color coding. (Green) Low expression; (red) high expression. SRF target muscle lineage genes or growth-related genes were assigned different colors (red or green). (B) The relative expression of known RhoA/MAL or Ras/ELK1 targets in MEFs at 30 min after serum stimulation was extracted from the microarray data. The expression levels of two sets genes in knockout MEFs were normalized to 1. (C) The relative expression of the two sets genes in wild-type, knockout, and KO+Med23 MEFs at 30 min after serum stimulation was analyzed by real-time PCR, with the expression in KO+Med23 MEFs normalized to 1. 
To further verify our hypothesis, the expression patterns of a larger number of well-documented RhoA/MAL (Actg2, Sm22a, My19, Cnn1, Acta1, Vcl, Acta2, and Crsp1) (Miano 2003) or Ras/ELK1 (Ier2, Krox20/Egr2, Junb, Egr3, Egr1, and Ier3) (Buchwalter et al. 2004) targets were extracted from our microarray data, and their relative expression did show opposite responses upon Med23 knockout (Fig. 3B), which could be verified by real-time PCR analysis (Fig. 3C). More strikingly, the expression of both of the two different sets of genes could be well rescued by reintroduction of $\mathrm{h} M e d 23$ (Fig. 3C), indicating the specific regulation of MED23 on their activity.

RhoA/MAL- and Ras/ELK1-activated genes constitute the most important and mutually exclusive two classes of SRF-controlled immediate-early genes in fibroblasts (Miano 2003; Posern and Treisman 2006). However, the mechanism by which the two sets of genes have a balanced expression pattern is not clear. Our previous data have shown that MED23 is required for Mediator recruitment to the Ras/ELK1 target gene promoters and their subsequent expression (Stevens et al. 2002; Wang et al. 2005), and our new observation showed that Med23 deficiency up-regulates the RhoA/MAL-controlled genes. Microarray analysis of the wild-type and Med23 knockout MEFs here further corroborated our observation. Thus, MED23 may function as a master modulator for the two sets of SRF target genes.

\section{Med23 controls a cell fate determination program between SMCs and adipocytes}

RhoA and MAL are involved in an efficient SMC differentiation program (Cen et al. 2004; Wang and Olson 2004; Mack and Hinson 2005; Pipes et al. 2006). In order to examine in detail the cellular consequence of Med23 depletion, we knocked down the expression of Med23 in C3H10T1/2 (10T1/2) cells, a mesenchymal stem cell line that is able to differentiate into numerous mesoderm cell types (Pinney and Emerson 1989). The MED23 protein was virtually undetectable in siMed23 10T1/2 cells (Fig. 4A, top). siCtrl and siMed23 10T1/2 cells were treated with an adipogenic cocktail to induce adipocyte differentiation (Huang et al. 2009; Wang et al. 2009). Consistent with our previous results in preadipocyte $3 \mathrm{~T} 3 \mathrm{~L} 1$ cells
A

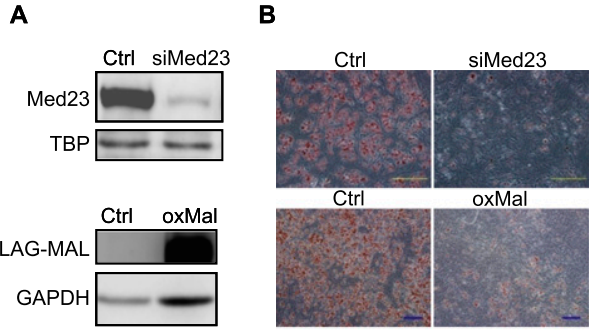

D
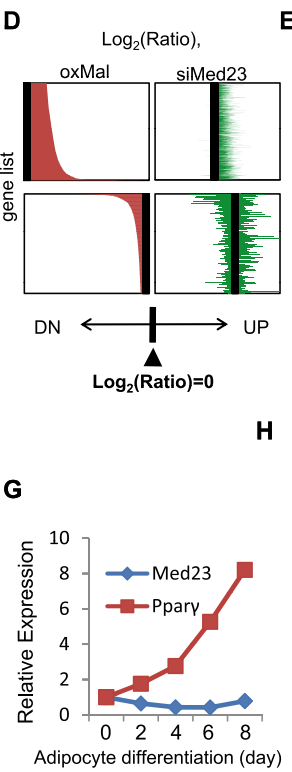

E
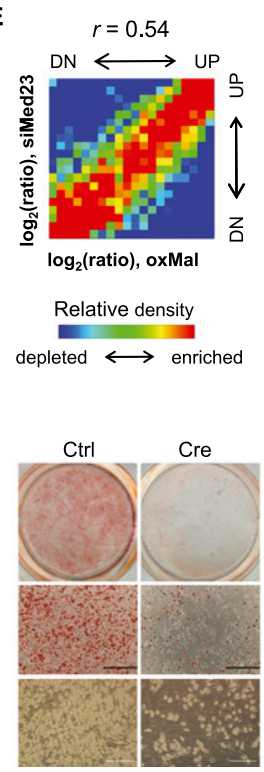

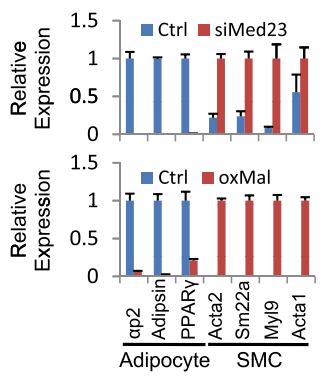

$\mathbf{F}$

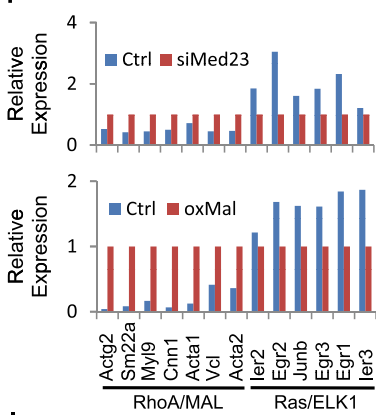

I

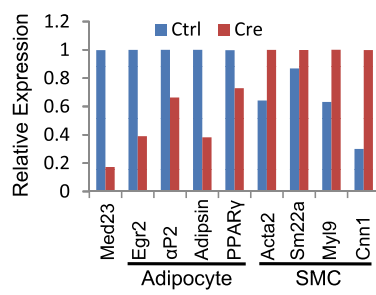

Figure 4. Analysis of lineage program and gene profiles regulated by MED23 and MAL. (A) Western blot was performed to confirm the siRNA depletion of endogenous MED23 and ectopic Flag-MAL expression in $10 \mathrm{~T} 1 / 2$ cells. TBP or GAPDH was blotted as a loading control. $(B)$ Ctrl, siMed23 (top panel), and oxMal (bottom panel) 10T1/2 cells were subjected to the hormone-induced adipocyte differentiation protocol. At day 8 post-induction, the cells were stained for lipid droplets with ORO. Bar, $200 \mu \mathrm{m} .(C)$ Realtime PCR analysis of adipocyte $(P P A R \gamma, \alpha p 2$, and Adipsin) and SMC (Acta2, Sm22a, My19, and Acta1) markers in Ctrl and siMed23 (top panel) or Ctrl and oxMal (bottom panel) $10 \mathrm{~T} 1 / 2$ cells at day 8 postinduction. Their expression was normalized to Gapdh. (D) oxMal-affected genes are shown in the left panel (top, up-regulated; bottom, down-regulated) according to the oxMal/Ctrl $\log _{2}$ ratio. The siMed23/ Ctrl $\log _{2}$ ratio of the same genes is listed in identical order in the right panel. $(E)$ Gene density map comparing gene expression changes in siMed23/Ctrl with those in oxMal/Ctrl. Genes were distributed in the table according to their expression ratios in the two comparisons. Spearman rank correlation coefficient $(r)$ is indicated. (UP) Up-regulated; (DN) down-regulated. More detail is offered in the Supplemental Material. (F) The expression pattern of known RhoA/MAL and Ras/ELK1 targets was extracted from microarray data. The expression in siMed23 (top panel) or oxMal (bottom panel) $10 \mathrm{~T} 1 / 2$ cells was normalized to $1 .(G)$ The mRNA levels of Med23 and PPAR $\gamma$ during adipocyte differentiation were analyzed using realtime PCR. The expression was normalized to $18 \mathrm{~S}$. (H) Ctrl ADSCs $\left(M e d 23^{\mathrm{fl} / \mathrm{fl}}\right)$ and Cre ADSCs $\left(\right.$ Med23 $3^{\mathrm{fl} / \mathrm{fl}}$ ADSCs infected with Cre-expressing adenovirus for 48 h) were subjected to the hormone-induced adipocyte differentiation protocol. At day 8 post-induction, ORO staining and bright-field pictures were taken. Bar, $200 \mu \mathrm{m}$. (I) The mRNA levels of Med23, adipocyte markers (Egr2/krox20, PPAR $\gamma, \alpha P 2$, and Adipsin), and SMC markers (Acta2, Sm22a, My19, and Cnn1) in Ctrl and Cre ADSCs were analyzed using real-time PCR at day 8 post-induction. The expression levels were normalized to $18 S$. 
(Wang et al. 2009), siMed23 10T1/2 cells were refractory to adipogenesis, as evidenced by Oil Red O (ORO) staining (Fig. 4B, top). Real-time PCR analysis revealed that the adipocyte markers $P P A R \gamma, \alpha P 2$, and Adipsin were ablated in siMed23 cells compared with siCtrl cells (Fig. 4C, top). Surprisingly, we observed a large increase of SMC markers such as Acta2, Sm22a, Myl9, and Acta1 in siMed23 cells (Fig. 4C, top). These results strongly suggest that Med23 antagonizes Mal by restricting SMC gene expression while promoting adipocyte gene expression during mesoderm stem cell differentiation.

To further investigate whether Mal indeed antagonizes Med23 in regulating the adipocyte lineage program, retroviral-mediated $\mathrm{Mal}$ overexpression (oxMal) was performed in 10T1/2 cells (Fig. 4A, bottom). oxMal greatly induced the expression of Acta2, Sm22a, Myl9, and Acta1 (Fig. 4C, bottom). Interestingly and expectedly, it greatly repressed adipocyte differentiation, as evidenced by ORO staining and real-time PCR analysis (Fig. 4B,C, bottom). Therefore, our results suggest Med23 and Mal antagonize each other in regulating the adipocyte and SMC programs.

To understand the mechanism of the antagonism between Med23 and Mal on a different cell lineage program, we compared the gene profiling changes resulting from oxMal or siMed23. oxMal led to 1678 up-regulated genes $\left(\log _{2}\right.$ ratio $\left.>0.7\right)$, and $1537(\sim 92 \%)$ of these genes were changed in the same direction by siMed23 $\left(\log _{2}\right.$ ratio $>0$ ) (Fig. 4D, top). Additionally, $\sim 78 \%$ (1185 of 1516) of oxMal down-regulated genes $\left(\log _{2}\right.$ ratio $\left.<-0.7\right)$ were also changed in the same direction by siMed23 $\left(\log _{2}\right.$ ratio < 0) (Fig. 4D, bottom). Moreover, global gene expression between siMed23/Ctrl and oxMal/Ctrl showed a correlation coefficient of 0.54 (Fig. 4E), suggesting a functional commonality in transcription control between these two manipulations. By looking into the detailed gene expression, effects similar to that observed in MEFs were also found in 10T1/2 cells: RhoA/MAL targets were upregulated in siMed23 cells, while Ras/ELK1 targets were down-regulated (Fig. 4F, top). Interestingly, besides greatly inducing RhoA target genes, oxMal also greatly repressed Ras/ELK1 targets (Fig. 4F, bottom), which might account for its repression effect on adipogenesis. In summary, the large overlapping changes in global gene expression resulting from oxMal and siMed23 explain the similar phenotype of siMed23 and oxMal in regulating the SMC and adipocyte lineage programs.

To further verify the in vivo function of Med23 in cell fate choice, we performed the following experiments in a possible adipocyte/SMC precursor, adipose-derived stem cells (ADSCs) isolated from mice, which can be induced to numerous cell types (Schaffler and Buchler 2007; de Villiers et al. 2009). While the SMC precursor is largely unknown, many new markers of white fat precursor have been identified recently, such as Sca1 (Rodeheffer et al. 2008; Tang et al. 2008). In order to specifically silence Med23 expression in $\mathrm{Sca}^{+}$ADSCs and avoid the offtarget effect of RNAi oligos, we used the Cre-loxp system. As the Med23 conditional knockout mice (Med23 $3^{\mathrm{fl} / \mathrm{fl}}$ ) were just generated by introducing loxP sites into the introns flanking Med23 exons 5-7, we isolated the Sca ${ }^{+}$ ADSCs from the Med $23^{\mathrm{fl} / \mathrm{fl}}$ mice following the literature (Rodeheffer et al. 2008), and then Cre-expressing adenovirus was used to infect the Med23 $3^{\mathrm{fl} / \mathrm{fl}} \mathrm{Scal}^{+}$ADSCs for inactivating Med23. The Med23 mRNA level in the adenovirus-infected (Cre) cells was reduced by $\sim 80 \%$ compared with that of the Ctrl cells (Fig. 4I). Both the Ctrl and $\mathrm{Cre} \mathrm{Sca} 1^{+}$populations were then exposed to an adipogenic hormone cocktail for $8 \mathrm{~d}$. The mRNA level of Med23 was not greatly changed, but the adipocyte marker $P P A R \gamma$ was progressively increased during adipocyte induction (Fig. 4G). However, Cre Scal ${ }^{+}$ADSCs accumulated far fewer lipids than the Ctrl population, as demonstrated by ORO staining and bright-field pictures (Fig. 4H). More strikingly, real-time PCR confirmed the attenuated expression of adipocyte markers but increased SMC markers in the Cre cells (Fig. 4I). Taken together, both the 10T1/2 and ADSC results supported the dual, opposing function of MED23 in fat and smooth muscle fate determination.

\section{Med23 deficiency facilitates mesenchymal stem cell differentiation into SMCs}

The promotion of SMC genes during adipocyte differentiation by siMed23 led us to directly investigate the function of Med23 during SMC differentiation. We first investigated whether siMed23 was sufficient to reprogram 10T1/2 into SMCs. Retrovirus-mediated Med23 knockdowns with two different oligos were both sufficient to guide $\sim 80 \%$ of $10 \mathrm{~T} 1 / 2$ cells into Acta2-positive cells (Supplemental Fig. S3A). SMC markers such as ACTA2, SM22A, CNN1, and CSRP1 were all increased in both knockdown cells (Supplemental Fig. S3B). MAL has been proved to be able to reprogram 10T1/2 into SMCs. (Wang et al. 2003). oxMal 10T1/2 cells exhibited a dramatic change in cell morphology. The SMC markers ACTA2, SM22A, and CSRP1 increased dramatically, as evidenced by immunostaining or Western blot analysis (Fig. 5A [cf. panels a and c], B [cf. lanes 1 and 3]). However, compared with oxMal, the induction of SMC genes in siMed23 10T1/2 cells was weaker, suggesting that depletion of Med23 alone is not sufficient to completely drive the process of SMC differentiation (Fig. 5B, cf. lanes 2 and 3). However, siMed23 greatly potentiated MAL's reprogramming effects. Combined treatment of oxMal and siMed23 resulted in robust SMC differentiation, significantly flattened and enlarged morphology, and greatly increased SMC gene expression compared with oxMal alone (Fig. 5A [panels d,h], B [lane 4]). The synergy between siMed23 and oxMal was also revealed by microarray analysis, where the expression of 768 out of the 867 co-up-regulated genes (by $\log _{2}$ ratio $>0.7$ ) were found to be altered to a greater extent by the combined treatment (Fig. 5C), which was confirmed by real-time PCR analysis of a list of cytoskeleton/SMC genes (Fig. 5D, top). Interestingly, 305 out of the 378 co-down-regulated genes (by $\log _{2}$ ratio $<-0.7$ ), including known Ras/ELK1 target genes (Egr1, Krox20/Egr2, Egr3, and c-fos), were synergistically repressed (Fig. 5C,D). 
A

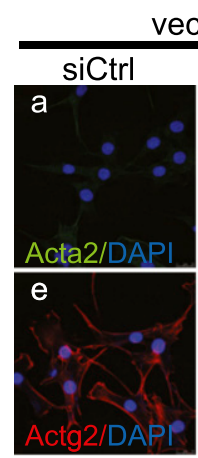

C

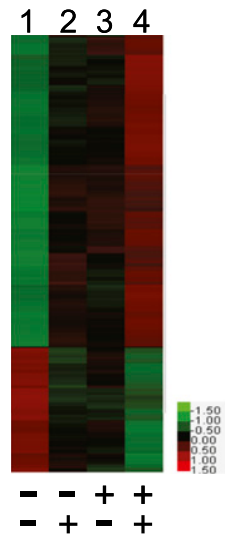

B

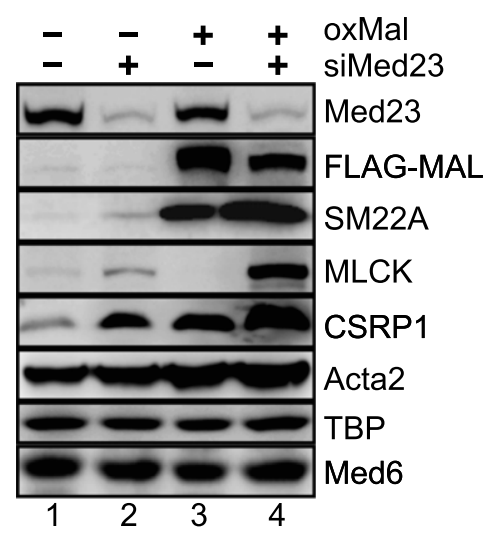

D

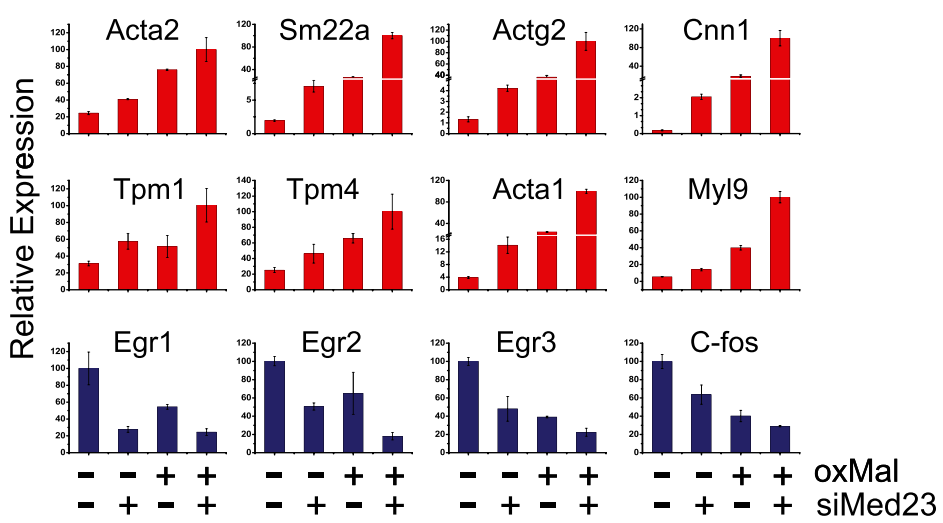

E

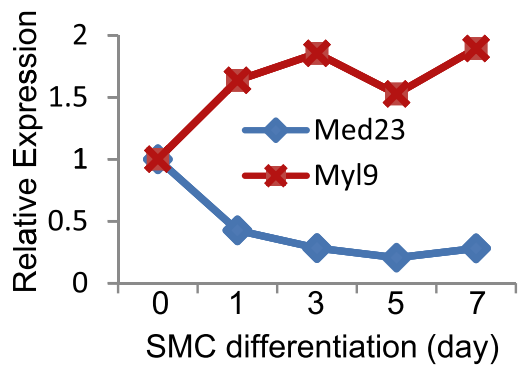

$\mathbf{F}$

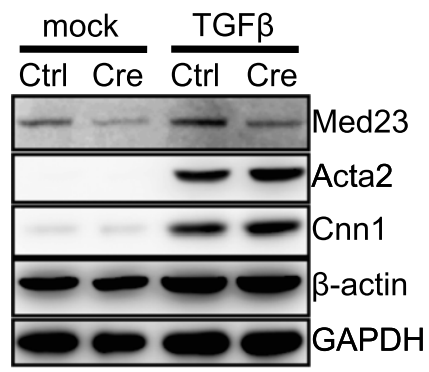

G
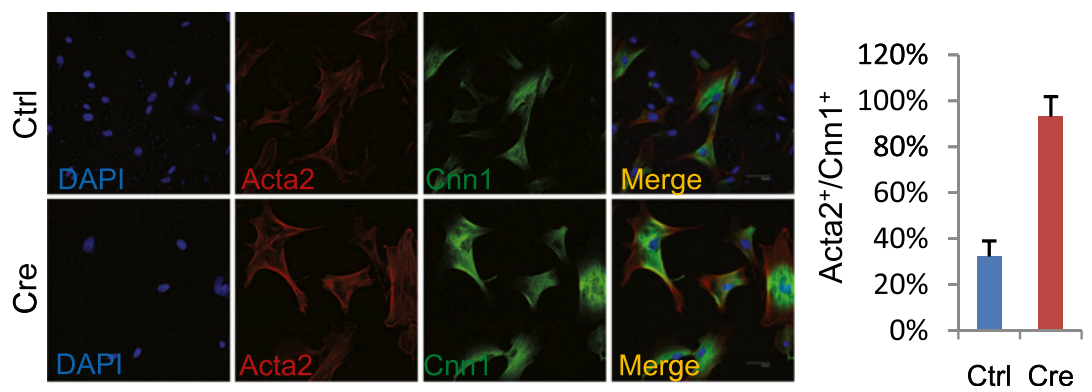

Figure 5. Med23 deficiency enhances SMC differentiation and gene expression. (A) Stable 10T1/2 cell lines were generated by retroviral expression of siMed23+oxMal. Ctrl, siMed23, oxMal, and siMed23+oxMal 10T1/2 cells were fixed and stained with DAPI, anti-ACTA2, and anti-ACTG2 antibodies. Bar, $25 \mu \mathrm{m}$. (B) Protein extracts from Ctrl, siMed23, oxMal, and siMed23+oxMal 10T1/2 cells were assayed by Western blot using the indicated antibodies. TBP and MED6 were blotted as loading controls. $(C)$ Heat map showing the synergy of siMed23 and oxMal on common target gene expression ( $\log _{2}$ ratio $\left.>0.7\right)$. More expression of common target genes was further changed in siMed23+oxMal than in siMed23 or oxMal 10T1/2 cells. (D) Total RNA from Ctrl, siMed23, oxMal, and siMed23+oxMal 10T1/2 cells were extracted and subjected to real-time PCR analysis of RhoA/MAL and Ras/ELK1 genes. The expression was normalized to Gapdh. (E) The mRNA levels of Med23 and Myl9 during SMC differentiation were analyzed using realtime PCR and normalized to 18S. (F) Ctrl and Cre ADSCs were treated with or without TGF $\beta$ for $24 \mathrm{~h}$. A Western blot was used to check the MED23 expression and the SMC marker ACTA2 and CNN1. $\beta$-Actin and GAPDH were used as loading controls. (G, left panel) Immunostaining of Ctrl and Cre ADSCs with the indicated antibodies. Bar, $50 \mu \mathrm{m}$. (Right panel) The percentage of ACTA2 and CNN1 double-positive cells was quantified relative to the total nuclei (from five random fields). 
Yin et al.

Primary ADSCs can be induced to SMCs under the proper culture conditions and TGF $\beta$ addition (Tang et al. 2008; Ball et al. 2010; Yalvac et al. 2010). We thus checked the function of Med23 during this process using the virally expressed Cre to delete Med23 from the ADSCs. The knockout efficiency of Med23 was confirmed by Western blot analysis (Fig. 5F). Interestingly, the mRNA level of Med23 progressively decreased during SMC differentiation, while the SMC marker Myl9 was progressively increased, suggesting a repressing function of Med23 during SMC differentiation (Fig. 5E). Remarkably, Cre ADSCs more efficiently differentiated into SMCs upon TGF $\beta$ treatment compared with Ctrl cells, as determined by the increased protein level of ACTA2 and CNN1 (Fig. 5F). Moreover, $\sim 90 \%$ of Cre cells were guided into the Acta $2^{+} / \mathrm{Cnn}^{+}$SMC lineage within $24 \mathrm{~h}$ of TGF $\beta$ treatment, whereas only $\sim 30 \%$ of Ctrl cells differentiated (Fig. 5G). Taken together, the results of both cell lines and primary cells strongly suggest that Med23 plays a repressing function during SMC differentiation.

\section{Med23 deficiency favors the formation of the $M A L-S R F$ complex over the ELK1-SRF complex}

To understand the mechanism by which Med23 deficiency promotes SMC genes expression, we performed a chromatin immunoprecipitation (ChIP) assay to examine the occupancy of related factors at SMC genes in $10 \mathrm{~T} 1 / 2$ cells. The ChIP analysis indicated that the depletion of Med23 enhanced the recruitment of Pol II to the promoter regions of Actg2, My19, and Acta2 (Fig. 6B; Supplemental Fig. S4A), which correlates with their upregulated transcription levels (Fig. 5D). MAL and ELK1 represent two exclusive transcription factors binding to SRF (Posern and Treisman 2006). First, we confirmed that the mRNA level of Mal and Elk1 was not significantly
A

B
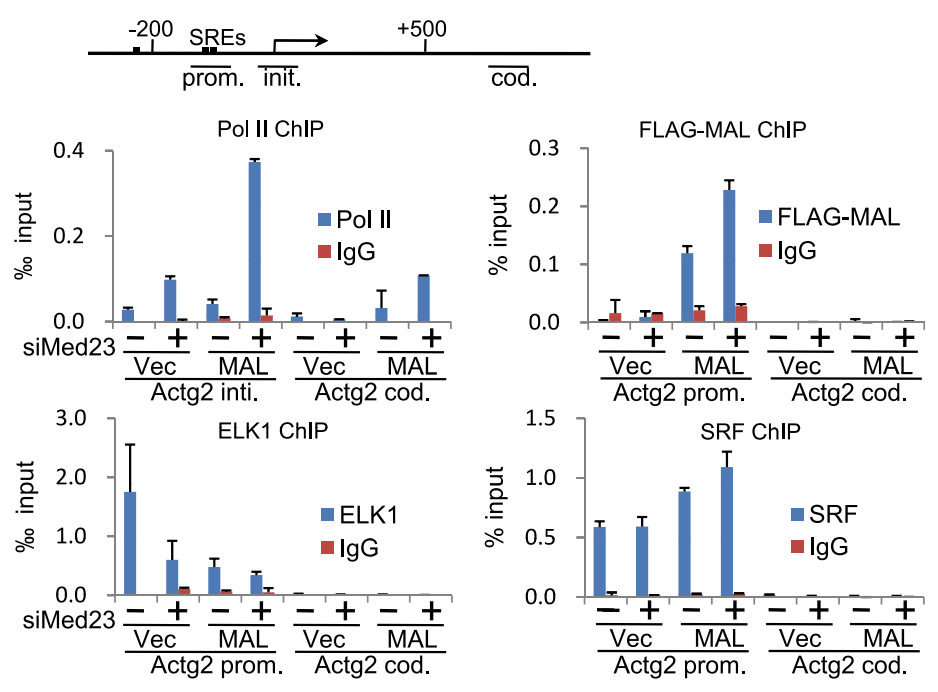

C

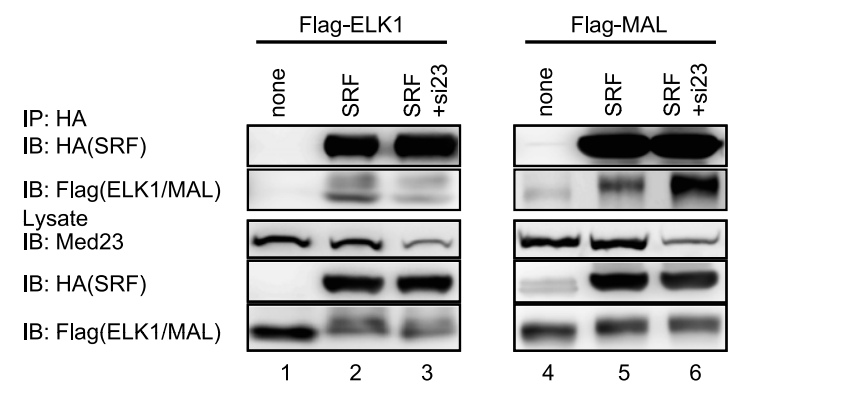

D

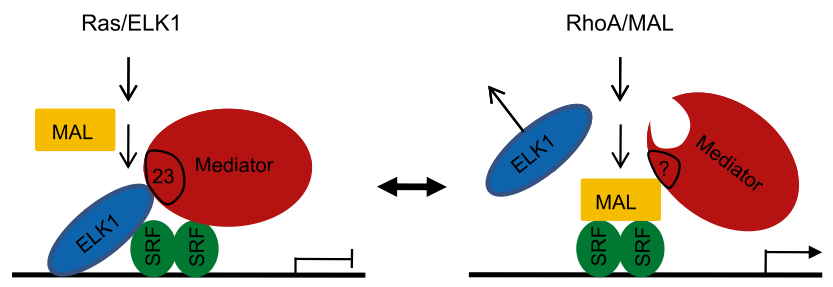

Cytoskeleton/SMC gene
Figure 6. MED23 modulates the regulator occupancy at the SMC genes. (A) SRF-binding sites. The line below shows the Actg2 gene region amplified in ChIP. (B) ChIP experiments were performed in Ctrl, siMed23, oxMal, and siMed23+oxMal 10T1/2 cells using antibodies against Pol II, Flag, SRF, and ELK1; IgG was used as a negative control. The precipitated DNA was analyzed by real-time PCR with primers targeting the Actg2 transcriptional initiation region (Actg2 init.), promoter region (Actg2 prom.), and coding region (Actg2 cod.). The relative binding level of each factor was calculated by normalization to the input DNA. The average of three separate experiments is shown, and the standard deviation is indicated. $(C)$ Co-IP of SRF with ELK1 (left panel) or MAL (right panel) in siCtrl and siMed23 HeLa cells. Flag-ELK1 (or FlagMAL) plasmid was transfected into HeLa cells with or without HA-SRF. Whole-cell extracts were analyzed directly (lysate) or following immunoprecipitation with anti-HA antibody (IP: HA). (D) A schematic model for Mediator MED23 in regulating the complex formation of SRF/ELK1 versus SRF/MAL. 
changed by Med23 depletion (Supplemental Fig. S4C). When Flag-MAL was ectopically expressed in 10T1/2 cells, Med23 depletion led to increased binding of FlagMAL and Pol II to SMC gene promoters as well as Pol II binding to their coding regions (Fig. 6B; Supplemental Fig. S4A). ELK1 has been shown to compete with Myocd for SRF binding to form an ELK1-SRF complex and antagonize the activation of SMC genes in vivo (Wang et al. 2004). We found that ELK1 repressed MAL-induced Sm22a reporter activity (Supplemental Fig. S4B), probably through a mechanism similar to that of ELK1 repressing Myocd activity (Wang et al. 2004). Strikingly, when Med23 was depleted, ELK1 binding to the Actg2 promoter was compromised and further decreased when siMed23 and oxMal were combined (Fig. 6B). Besides the transcription factors MAL and ELK1, SRF is also a necessary cofactor for cytoskeleton/SMC gene expression. As mentioned, the expression of SRF was slightly increased in Med23 knockout MEFs, but the modest increase is not enough to explain the great up-regulation of cytoskeleton/SMC genes. To further address the question of whether MED23 affects the recruitment of SRF, we performed ChIP and ChIP-seq (ChIP combined with deep sequencing) experiments to compare the SRF-binding profile in siCtrl and siMed23 10T1/2 cells. SRF occupancy at the global gene promoter region was slightly increased by Med23 depletion, whereas its binding intensity in the gene body was not altered (Supplemental Fig. S4D). For siMed23-upregulated genes, the SRF occupancy pattern was similar to that of the whole genome region, slightly increased at the promoter but not changed at the gene body, which is also consistent with our ChIP analysis of the Actg2 gene (Fig. 6B; Supplemental Fig. S4D). For the siMed23-downregulated genes, the SRF occupancy at both the promoter and the gene body region was not significantly changed (Supplemental Fig. S4D). Considering that MED23 interacts directly with ELK1 and is required for its activity (Wang et al. 2009), these data suggest that Med23 depletion destabilized ELK1 binding to the SMC gene promoters, thus promoting MAL and SRF binding to activate cytoskeleton/SMC gene expression.

The interaction between ELK1/SRF and MAL/SRF (activator/cofactor) is important for their transcription activity. Considering the well-known function of the Mediator complex in bridging activators and general transcription factors, we wanted to know whether the Mediator complex with or without MED23 differentially affected the complex formation of ELK1/SRF versus MAL/SRF. We performed a coimmunoprecipitation (co-IP) experiment by cotransfecting HA-tagged SRF with either Flag-tagged MAL or ELK1 into HeLa cells. HA pull-down followed by Western blot revealed that Med23 depletion resulted in reduced Flag-ELK1 signal (Fig. 6C, cf. lanes 2 and 3) but enhanced Flag-MAL signal (Fig. 6C, cf. lanes 5 and 6), while SRF expression remains at similar levels.

Taken together, these data suggest that the presence of MED23 favors ELK1-SRF complex formation at the cytoskeleton/SMC gene promoters. In contrast, the absence of MED23 destabilizes ELK1 binding and MALSRF complex formation at cytoskeleton/SMC promoters
(Fig. 6D). Therefore, our biochemical data are consistent with the effect that the presence of MED23 facilitates the expression of ELK1-driven growth/adipogenic genes, but the absence of MED23 enhances the expression of cytoskeleton/SMC genes driven by MAL.

\section{Role of Med23 in zebrafish smooth muscle development}

The zebrafish MED23 protein sequence shares $90 \%$ and $89 \%$ identity with those of human and mouse MED23, respectively. We also investigated the in vivo function of Med23 during zebrafish development. Two morpholinos (MOs) were designed to specifically block the translation of Med23 mRNAs, and the effect was verified by Western blot analysis (Fig. 7A). Interestingly, developmental abnormalities were observed in both morphants, such as shortened body length, a ventrally curved tail, heart edema, and a lower heart rate (Fig. 7B; Supplemental Movie S1). In addition, depletion of Med23 in the vascular Tg(Flk1:GFP) (Cross et al. 2003) transgenic fish caused a significant increase of the fluorescence intensity in the axial vessels and heart region (Fig. 7C), suggesting the function of Med23 in repressing the hypertrophy of cardiovascular system. The role of MED23 in adipogenesis could not be assessed here because the adipocytes have not been described in teleosts so far. SMC developmental marker genes were analyzed using real-time PCR and wholemount in situ hybridization. By $48 \mathrm{~h}$ post-fertizilation (hpf), the SMC marker genes Acta2, Sm22a, Myh11, and Myl9 were up-regulated in Med23 morphants (Fig. 7D,E). Collectively, Med23 deficiency promotes the precocious development of vascular smooth muscle, suggesting its requirement for the proper development of the cardiovascular system in zebrafish.

\section{Discussion}

A long-standing issue in the field of SRF transcriptional regulation concerns the mechanisms responsible for the apparent antagonistic interplay between the SRF targeted growth-related and muscle-related genes (Posern and Treisman 2006). Our study demonstrated that the balance of these two types of SRF target genes, driven by MAL or ELK1, can be modulated by the presence or absence of the Mediator MED23 subunit. This observation is highly significant because the Ras/ELK1 and RhoA/MAL pathways need to be properly balanced for normal cellular function and development.

We previously found that Med23 depletion prevents adipogenesis, which instigated our curiosity and led to a question: What is the consequent cell fate when Med23 is depleted from the cell? Initially, we observed that the Med $23^{-1-}$ fibroblasts displayed a flattened and enlarged morphology with enhanced stress fiber and focal adhesion formation. Transcriptome analysis revealed that Med23 deficiency resulted in opposite responses of the seruminduced Ras/ELK1 target genes (down-regulated) versus RhoA/MAL target genes (up-regulated). We then went on to demonstrate the direct effect of Med23 deficiency on 
Yin et al.

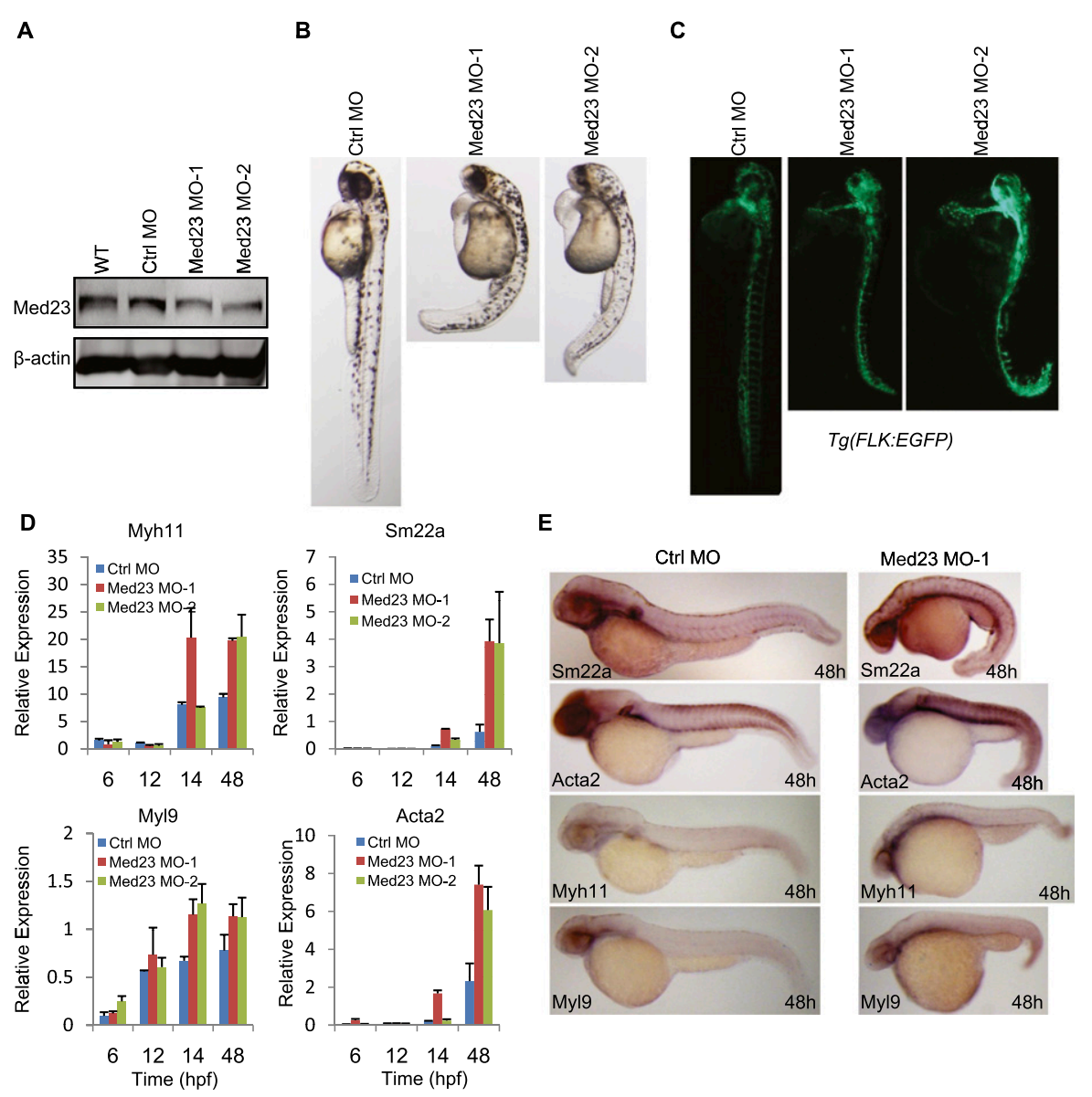

Figure 7. Med23 deficiency promotes smooth muscle development in zebrafish. (A) Western blot analysis of MED23 knockdown efficiency. Embryos without injection (wild-type [WT], lane 1), injected with $4 \mathrm{ng}$ of Ctrl MO (lane 2), 4 ng of Med23 MO-1 (lane 3), or $2 \mathrm{ng}$ of Med23 MO-2 (lane 4) were harvested at 24 hpf. Protein samples were prepared, subjected to SDS-PAGE, and blotted with antibody against Med23. $\beta$-Actin was used as a loading control. (B) Phenotypic analysis of zebrafish embryos at 48 hpf. Embryos injected with Ctrl MO, Med23 MO-1, or Med23 MO-2 were analyzed. Embryos are lateral views with anterior to the top. (C) Embryos derived from the transgenic zebrafish line $\mathrm{Tg}(f l k: E G F P)$ were injected with the two Med23 MOs and showed significant increased fluorescent intensity. Embryos are lateral views with anterior to the top. (D) Real-time PCR analysis of SMC markers (Acta2, Sm22a, Myh11, and Myl9) at the indicated time points. (E) Whole-mount in situ hybridization for embryos injected with Ctrl MO and Med23 MO-1 was performed at 48 hpf. Riboprobes to SMC markers (Sm22a, Acta2, Myh11, and My19) were used. All embryos are lateral views with anterior to the left.

ELK1 and MAL function using a battery of biochemical assays. Specifically, we found that (1) Med23 depletion decreased ELK1 binding to SMC promoters but increased MAL and SRF binding to SMC promoters, as shown by the ChIP assays (Fig. 6B; Supplemental Fig. S4A), and (2) Med23 depletion enhanced MAL-SRF interaction but reduced ELK1-SRF interaction, as shown by the co-IP experiment (Fig. 6C). These studies suggest a molecular model in which the presence of MED23 favors ELK1-SRF complex formation, which in turn activates the growthrelated genes and represses the cytoskeleton/SMC genes; in contrast, the absence of MED23 destabilizes the ELK1SRF complex and decreases the expression of growth/ adipogenic genes but promotes the MAL-SRF complex formation at SMC genes to induce their activation. A key question here is: How does MED23 regulate SRF/ELK1 complex formation to compete with MAL/SRF complex formation? Our previous work demonstrated that ELK1 interacts with MED23 in vitro and in vivo, and the transcription activity of ELK1 was abolished in $\mathrm{Med}_{23} \mathrm{3}^{-/-}$ cells and can be restored by re-expressing MED23 (Stevens et al. 2002; Wang et al. 2009); ELK1 can recruit Mediator in a MED23-dependent manner, as revealed by the ChIP assay (Wang et al. 2005, 2009). Moreover, increasing the dosage of transfected Elk1 inhibited the expression of the MAL-driven Sm22a luciferase reporter. These observations suggest that perhaps the Mediator complex with MED23 enhances ELK1/SRF complex formation through a cooperative binding mechanism to favor growth/adipogenic gene expression, which consequently facilitates the competition of ELK1 with MAL for forming a complex with SRF (ELK1/SRF) to inhibit cytoskeleton/SMC gene expression. Strikingly and consistent with our model, a recent study found that a Med23 point mutation linked with a 
familial intellectual disability is able to reduce ELK1 occupancy at the growth-related immediate-early gene promoters in the skin fibroblasts isolated from a family member with this genetic mutation (Hashimoto et al. 2011).

In this study, we also excluded a number of alternative molecular mechanisms linking Med23 deficiency with cytoskeleton/SMC gene regulation. For example, we demonstrated that Med23 deficiency (1) did not alter RhoA signal transduction and MAL transcriptional activity and (2) did not modulate the SRF level to up-regulate the cytoskeleton genes; furthermore, (3) many other Mediator components, including the repressive submodule CDK8/MED12, failed to change the cytoskeleton gene expression.

To follow up on these molecular mechanism studies, we performed cell differentiation experiments and revealed that Med23-depleted mesenchymal stem cells (10T1/2 and ADSCs) are prone to differentiation into SMCs but are refractory to differentiation into adipocytes. Remarkably, using zebrafish, we showed that Med23 deficiency promotes abnormal development of the vascular smooth muscle. Interestingly and significantly, while ectopic expression of Elk1 inhibits RhoA/MAL target SMC genes, Mal overexpression attenuated adipocyte gene expression and adipogenesis in mesenchymal stem cells (Fig. 4B,C). Overall, MED23 acts as a restrictive regulator of RhoA/MAL signaling and SMC differentiation while functioning as a positive regulator for Ras/ELK1 targeted genes and adipocyte differentiation. Our study suggested that MED23 controls the dynamic balance of the two pathways, thus providing an in-depth mechanism by which these two pathways are modulated to ensure precise molecular/cellular output.

The potential lineage relationship between SMCs and adipocytes is an outstanding question in developmental biology. According to the literature, adipocytes have been shown to arise from the pericytes or endothelial cells of the vasculature (Tang et al. 2008; Gupta et al. 2012; Tran et al. 2012), and certainly both the adipocytes and SMCs are derived from the mesoderm cells. There is a good possibility that some kinds of vasculature cells are able to differentiate into either adipocytes or SMCs. However, at the present time, there is no report of an in vivo lineage tracing experiment demonstrating a "common precursor" for both fat cells and SMCs. Once the molecular marker for a "common precursor" is available in the future, we should have an in vivo model in which we could manipulate Med23 level and then show its effect on adipocyte/ SMC differentiation by using our conditional knockout mice. Although the lineage tracing of SMC and adipocytes has not been accomplished at the present time, our analysis of the primary ADSCs with Med23 depletion unequivocally demonstrated that Med23 plays a positive role in adipocyte differentiation while playing a negative role in SMC differentiation, which leads to our overall conclusions that Mediator Med23 plays a "Ying-Yang" role in directing SMC and adipocyte differentiation.

Last, given that the imbalance of fat and smooth muscle tissue is involved in many metabolic and cardiovascular diseases such as obesity, diabetes, atherosclero- sis, and hypertension, our findings on the "Ying-Yang" function of MED23 may provide a better understanding of and a possible therapeutic target for these diseases.

\section{Materials and methods}

Mice

Med23 conditional knockout mice were generated according to standard gene targeting protocols. In brief, exons 5-7 were flanked by two loxP sites, and a frt-Neo-frt cassette was used as a positive selection marker. The embryonic stem cells used for gene targeting were derived from the Sv129 strain. Chimeric mice were generated by injecting embryonic stem cells into C57BL/6 blastocysts followed by transfer to pseudopregnant mice. $M e d 23^{\mathrm{fl} / \mathrm{fl}}$ mice were maintained in a mixed genetic background of C57BL/6:Sv129.

\section{Cell culture}

Wild-type and Med23 knockout MEFs were isolated from 9.5-d embryos and self-immortalized from a standard protocol (Balamotis et al. 2009; Wang et al. 2009). The ADSCs were isolated from inguinal white adipose depots as described (Tang et al. 2008) and subjected to FACS or plated in a 24-well tissue culture dish. MEF, 10T1/2, HeLa, and Cos7 cells were maintained in Dulbecco's modified Eagle's medium (DMEM) containing $10 \%$ FBS (Hyclone). The isolated ADSCs were maintained in DMEM/F12 (1:1) with 10\% FBS (Hyclone). ADSCs between passages 2 and 5 were used in the experiments.

\section{Retrovirus and adenovirus infection}

Using retrovirus to establish stable cell lines to knock down or overexpress a gene of interest was based on the manufacturer's recommendation (Clontech). Retroviruses were generated following the cotransfection of recombinant pSiren-RetroQ (for knockdown) or pMSCV-hygro (for overexpression) plasmid with pCL10A1 helper plasmid into 293T cells using Lipofectamin 2000 (Invitrogen). 293T culture supernatants containing retroviruses were harvested $48 \mathrm{~h}$ later, supplemented with $20 \mu \mathrm{g} / \mathrm{mL}$ polybrene, passed through a $0.45-\mu \mathrm{m}$ filter, and then added to the intended cells for a centrifugation at $2500 \mathrm{rpm}$ for $1.5 \mathrm{~h}$ at $30^{\circ} \mathrm{C}$. Twenty-four hours after spin infection, MEFs were selected with $50 \mu \mathrm{g} / \mathrm{mL}$ puromycin (Sigma-Aldrich), and 10T1/2 cells were selected with $10 \mu \mathrm{g} / \mathrm{mL}$ puromycin or $50 \mu \mathrm{g} / \mathrm{mL}$ hygromycin. See the Supplemental Material for RNAi oligonucleotide sequences. Cre-expressing adenoviruses were generated following the manufacturer's recommendation (Qbiogene). For adenoviral infection of ADSCs, 30\% confluent cell cultures were incubated with the Cre-expressing adenovirus (multiplicity of infection, 100) for $48 \mathrm{~h}$ in complete growth medium. After $48 \mathrm{~h}$ of incubation, the medium was replaced, and cells were carried further for various experiments.

\section{Adipocyte and SMC differentiation}

$10 \mathrm{~T} 1 / 2$ cells were plated at a density of $5 \times 10^{4}$ per $3.5-\mathrm{cm}$ dish in DMEM cell culture medium with $10 \%$ FBS (Hyclone) and $10 \mu \mathrm{g} / \mathrm{L}$ BMP4 (R\&D Systems). Two days after the cells reached confluence, the cells were treated with culture medium containing differentiation cocktail $15 \mathrm{mg} / \mathrm{L}$ insulin, $0.5 \mathrm{mM}$ IBMX [3-isobutyl-1-methylxanthine], $1 \mu \mathrm{M}$ dexamethasone, $0.5 \mathrm{mg} / \mathrm{L}$ troglitazone; all from Sigma-Aldrich). After $48 \mathrm{~h}$, the cells were switched to medium containing $5 \mathrm{mg} / \mathrm{L}$ insulin, and the medium 
was replenished every $2 \mathrm{~d}$. For adipocyte differentiation, $S c a 1^{+}$ ADSCs were induced by the three-hormone cocktail similar to the 3T3-L1 induction. For SMC differentiation, $5 \times 10^{4}$ lowpassage ADSCs were seeded on coverslips (Fisher Scientific) in 12 -well dishes and exposed to medium with reduced serum (0.5\% FBS) supplemented with $10 \mathrm{ng} / \mathrm{mL}$ TGF $\beta$ (R\&D Systems). The medium was changed every $2 \mathrm{~d}$.

\section{Microarray analysis}

Mouse genome-wide gene expression analysis was performed using Affymetrix Mouse Gene 4302.0 array. Six arrays were probed with cDNA synthesized from total RNA isolated from wild-type and Med23 knockout MEF cells treated with or without $15 \%$ FBS serum stimulation as described above. Four arrays were probed with cDNA synthesized from total RNA isolated from siCtrl, siMed23, oxMal, and siMed23+oxMal 10T1/2 cells.

\section{ChIP, ChIP-seq, and data analysis}

ChIP assays were performed as described previously (Wang et al. 2005), except that the immunoprecipitated DNA were quantified using real-time PCR. The primers for analyzing the ChIP DNA are provided in the Supplemental Material. For ChIP-seq, the immunoprecipitated DNA concentration was measured by Qubit Fluorometer (Invitrogen). DNA was purified using ChIP-seq sample prep kit (Illumina) and subjected to 76 bases of sequencing on Genome Analyzer IIx (Illumina). See the Supplemental Material for more detail.

\section{Accession numbers}

Microarray and ChIP-seq data in this study are available for download from Gene Expression Omnibus (GEO) (http://www. ncbi.nlm.nih.gov/geo). The GEO accession ID for the microarray data is GSE40286 and the accession ID for the ChIP-seq data is GSE40369.

\section{Acknowledgments}

We thank Drs. Ron Prywes and Arnie Berk for generously providing reagents, and Drs. Naihe Jing, Ping Hu, Yi Zeng, and Ying Jin for helpful suggestions. This work was supported in part by grants from CAS (XDA01010401), China MOST (2009CB941100 and 2011CB510104), and CNSF (81030047) (to G.W.), and from the NIH (GM084089 and DK094207) (to B.T.). G.W. is a scholar of the "Hundred Talent Program."

\section{References}

Balamotis MA, Pennella MA, Stevens JL, Wasylyk B, Belmont AS, Berk AJ. 2009. Complexity in transcription control at the activation domain-mediator interface. Sci Signal 2: ra20. doi: 10.1126/scisignal.1164302.

Ball SG, Shuttleworth CA, Kielty CM. 2010. Platelet-derived growth factor receptors regulate mesenchymal stem cell fate: Implications for neovascularization. Expert Opin Biol Ther 10: $57-71$.

Blazek E, Mittler G, Meisterernst M. 2005. The mediator of RNA polymerase II. Chromosoma 113: 399-408.

Boyer TG, Martin ME, Lees E, Ricciardi RP, Berk AJ. 1999. Mammalian Srb/Mediator complex is targeted by adenovirus E1A protein. Nature 399: 276-279.

Buchwalter G, Gross C, Wasylyk B. 2004. Ets ternary complex transcription factors. Gene 324: 1-14.
Cen B, Selvaraj A, Prywes R. 2004. Myocardin/MKL family of SRF coactivators: Key regulators of immediate early and muscle specific gene expression. J Cell Biochem 93: 74-82.

Cross LM, Cook MA, Lin S, Chen JN, Rubinstein AL. 2003. Rapid analysis of angiogenesis drugs in a live fluorescent zebrafish assay. Arterioscler Thromb Vasc Biol 23: 911-912.

de Villiers JA, Houreld N, Abrahamse H. 2009. Adipose derived stem cells and smooth muscle cells: Implications for regenerative medicine. Stem Cell Rev 5: 256-265.

Ding N, Zhou H, Esteve PO, Chin HG, Kim S, Xu X, Joseph SM, Friez MJ, Schwartz CE, Pradhan S, et al. 2008. Mediator links epigenetic silencing of neuronal gene expression with X-linked mental retardation. Mol Cell 31: 347-359.

Ge K, Guermah M, Yuan CX, Ito M, Wallberg AE, Spiegelman BM, Roeder RG. 2002. Transcription coactivator TRAP220 is required for PPAR $\gamma 2$-stimulated adipogenesis. Nature 417: 563-567.

Gineitis D, Treisman R. 2001. Differential usage of signal transduction pathways defines two types of serum response factor target gene. J Biol Chem 276: 24531-24539.

Gupta RK, Mepani RJ, Kleiner S, Lo JC, Khandekar MJ, Cohen P, Frontini A, Bhowmick DC, Ye L, Cinti S, et al. 2012. Zfp423 expression identifies committed preadipocytes and localizes to adipose endothelial and perivascular cells. Cell Metab 15: 230-239.

Hashimoto S, Boissel S, Zarhrate M, Rio M, Munnich A, Egly JM, Colleaux L. 2011. MED23 mutation links intellectual disability to dysregulation of immediate early gene expression. Science 333: 1161-1163.

Hong JH, Hwang ES, McManus MT, Amsterdam A, Tian Y, Kalmukova R, Mueller E, Benjamin T, Spiegelman BM, Sharp PA, et al. 2005. TAZ, a transcriptional modulator of mesenchymal stem cell differentiation. Science 309: 1074-1078.

Huang H, Song TJ, Li X, Hu L, He Q, Liu M, Lane MD, Tang QQ. 2009. BMP signaling pathway is required for commitment of C3H10T1/2 pluripotent stem cells to the adipocyte lineage. Proc Natl Acad Sci 106: 12670-12675.

Kajimura S, Seale P, Kubota K, Lunsford E, Frangioni JV, Gygi SP, Spiegelman BM. 2009. Initiation of myoblast to brown fat switch by a PRDM16-C/EBP- $\beta$ transcriptional complex. Nature 460: 1154-1158.

Kato Y, Habas R, Katsuyama Y, Naar AM, He X. 2002. A component of the ARC/Mediator complex required for TGFß/Nodal signalling. Nature 418: 641-646.

Komori T. 2002. [Cbfa1/Runx2, an essential transcription factor for the regulation of osteoblast differentiation]. Nihon Rinsho 60: 91-97.

Mack CP, Hinson JS. 2005. Regulation of smooth muscle differentiation by the myocardin family of serum response factor co-factors. J Thromb Haemost 3: 1976-1984.

Malik S, Roeder RG. 2010. The metazoan Mediator co-activator complex as an integrative hub for transcriptional regulation. Nat Rev Genet 11: 761-772.

Miano JM. 2003. Serum response factor: Toggling between disparate programs of gene expression. I Mol Cell Cardiol 35: 577-593.

Miralles F, Posern G, Zaromytidou AI, Treisman R. 2003. Actin dynamics control SRF activity by regulation of its coactivator MAL. Cell 113: 329-342.

Olson EN. 1990. MyoD family: A paradigm for development? Genes Dev 4: 1454-1461.

Philippar U, Schratt G, Dieterich C, Muller JM, Galgoczy P, Engel FB, Keating MT, Gertler F, Schule R, Vingron $M$, et al. 2004. The SRF target gene Fhl2 antagonizes RhoA/MALdependent activation of SRF. Mol Cell 16: 867-880. 
Pinney DF, Emerson CP Jr. 1989. 10T1/2 cells: An in vitro model for molecular genetic analysis of mesodermal determination and differentiation. Environ Health Perspect 80: 221-227.

Pipes GC, Creemers EE, Olson EN. 2006. The myocardin family of transcriptional coactivators: Versatile regulators of cell growth, migration, and myogenesis. Genes Dev 20: 15451556.

Posern G, Treisman R. 2006. Actin' together: Serum response factor, its cofactors and the link to signal transduction. Trends Cell Biol 16: 588-596.

Rodeheffer MS, Birsoy K, Friedman JM. 2008. Identification of white adipocyte progenitor cells in vivo. Cell 135: 240249.

Schaffler A, Buchler C. 2007. Concise review: Adipose tissuederived stromal cells-basic and clinical implications for novel cell-based therapies. Stem Cells 25: 818-827.

Seale P, Bjork B, Yang W, Kajimura S, Chin S, Kuang S, Scime A, Devarakonda S, Conroe HM, Erdjument-Bromage H, et al. 2008. PRDM16 controls a brown fat/skeletal muscle switch. Nature 454: 961-967.

Settleman J. 2003. A nuclear MAL-function links Rho to SRF. Mol Cell 11: 1121-1123.

Stevens JL, Cantin GT, Wang G, Shevchenko A, Shevchenko A, Berk AJ. 2002. Transcription control by E1A and MAP kinase pathway via Sur2 mediator subunit. Science 296: 755-758.

Takahashi K, Yamanaka S. 2006. Induction of pluripotent stem cells from mouse embryonic and adult fibroblast cultures by defined factors. Cell 126: 663-676.

Tang W, Zeve D, Suh JM, Bosnakovski D, Kyba M, Hammer RE, Tallquist MD, Graff JM. 2008. White fat progenitor cells reside in the adipose vasculature. Science 322: 583-586.

Tontonoz P, Hu E, Spiegelman BM. 1995. Regulation of adipocyte gene expression and differentiation by peroxisome proliferator activated receptor $\gamma$. Curr Opin Genet Dev 5: 571-576.

Tran KV, Gealekman O, Frontini A, Zingaretti MC, Morroni M, Giordano A, Smorlesi A, Perugini J, De Matteis R, Sbarbati A, et al. 2012. The vascular endothelium of the adipose tissue gives rise to both white and brown fat cells. Cell Metab 15: 222-229.

Van Aelst L, D'Souza-Schorey C. 1997. Rho GTPases and signaling networks. Genes Dev 11: 2295-2322.

Vartiainen MK, Guettler S, Larijani B, Treisman R. 2007. Nuclear actin regulates dynamic subcellular localization and activity of the SRF cofactor MAL. Science 316: 17491752.

Wang DZ, Olson EN. 2004. Control of smooth muscle development by the myocardin family of transcriptional coactivators. Curr Opin Genet Dev 14: 558-566.

Wang Z, Wang DZ, Pipes GC, Olson EN. 2003. Myocardin is a master regulator of smooth muscle gene expression. Proc Natl Acad Sci 100: 7129-7134.

Wang Z, Wang DZ, Hockemeyer D, McAnally J, Nordheim A, Olson EN. 2004. Myocardin and ternary complex factors compete for SRF to control smooth muscle gene expression. Nature 428: 185-189.

Wang G, Balamotis MA, Stevens JL, Yamaguchi Y, Handa H, Berk AJ. 2005. Mediator requirement for both recruitment and postrecruitment steps in transcription initiation. Mol Cell 17: 683-694.

Wang W, Huang L, Huang Y, Yin JW, Berk AJ, Friedman JM, Wang G. 2009. Mediator MED23 links insulin signaling to the adipogenesis transcription cascade. Dev Cell 16: 764771.

Wernig M, Lengner CJ, Hanna J, Lodato MA, Steine E, Foreman R, Staerk J, Markoulaki S, Jaenisch R. 2008. A drug-inducible transgenic system for direct reprogramming of multiple somatic cell types. Nat Biotechnol 26: 916-924.

Yalvac ME, Ramazanoglu M, Rizvanov AA, Sahin F, Bayrak OF, Salli U, Palotas A, Kose GT. 2010. Isolation and characterization of stem cells derived from human third molar tooth germs of young adults: Implications in neo-vascularization, osteo-, adipo- and neurogenesis. Pharmacogenomics $I$ 10: 105-113. 


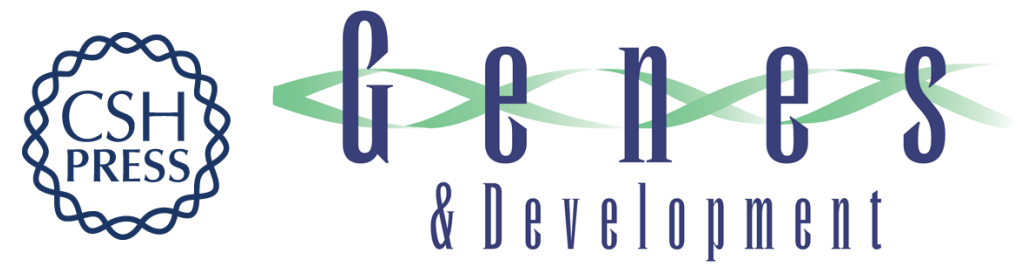

\section{Mediator MED23 plays opposing roles in directing smooth muscle cell and adipocyte differentiation}

Jing-wen Yin, Yan Liang, Ji Yeon Park, et al.

Genes Dev. 2012, 26: originally published online September 12, 2012

Access the most recent version at doi:10.1101/gad.192666.112

\section{Supplemental http://genesdev.cshlp.org/content/suppl/2012/09/05/gad.192666.112.DC1 Material}

References This article cites 45 articles, 13 of which can be accessed free at: http://genesdev.cshlp.org/content/26/19/2192.full.html\#ref-list-1

\section{License}

Email Alerting

Receive free email alerts when new articles cite this article - sign up in the box at the top Service 